Songül Ünüvara, Gözde Girgin, Ş. Sezin Palabıyık ${ }^{\mathrm{b}}$, Tülay Karaağaç, Osman Ilhami Özcebe, Gönül Şahin' and Terken Baydar*

\title{
Spot analyses of serum neopterin, tryptophan and kynurenine levels in a random group of blood donor population
}

\begin{abstract}
In transfusions, safety measures are used for the health of both receivers and donors. In this regard, early and sensitive markers are strongly needed. The aim of this study was to evaluate neopterin profile and tryptophan degradation in a group of Turkish blood donors. The effect of age, gender, blood type and Rh factor were also assessed. Neopterin was measured by ELISA kits. Serum tryptophan and kynurenine were determined by high performance liquid chromatography. Kynurenine/tryptophan (kyn/ trp) was calculated to estimate tryptophan degradation. Tryptophan, kynurenine and kyn/trp levels were found to be higher in males compared with females (all $\mathrm{p}<0.05$ ). Neopterin and kynurenine levels tended to increase with age in age subgroups (all $\mathrm{p}<0.05$ ). On the contrary, smoking, $\mathrm{Rh}$ factor and blood types were all observed to have no effect on measured parameters (all $p>0.05$ ). In routine analysis, early and reliable biomarkers are needed. In parallel with current markers, neopterin can be used to select safe donors and to maintain the safety of transfusion. In the face of our results, we recommend that donations with neopterin levels above $19 \mathrm{nmol} / \mathrm{L}$ should be excluded from the program because increased neopterin levels indicate activation of the cellular immune system.
\end{abstract}

Keywords: blood bank; indoleamine-2,3-dioxigenase; kynurenine; neopterin; tryptophan.

aPresent address: Faculty of Pharmacy, Toxicology Department, İnönü University, Malatya, Turkey.

bPresent address: Faculty of Pharmacy, Toxicology Department, Hacettepe University, Ankara, Turkey.

cPresent address: Faculty of Pharmacy, Toxicology Department, Eastern Mediterranean University, Famagusta, North Cyprus.

*Corresponding author: Prof. Dr. Terken Baydar, Faculty of Pharmacy, Toxicology Department, Hacettepe University, 06100 Sihhiye, Ankara, Turkey, E-mail: tbaydar@hacettepe.edu.tr

Songül Ünüvar, Gözde Girgin and Gönül Şahin: Faculty of Pharmacy, Toxicology Department, Hacettepe University, Ankara, Turkey Ş. Sezin Palabıyık: Faculty of Pharmacy, Toxicology Department, Atatürk University, Erzurum, Turkey

Tülay Karaağaç and Osman Ithami Özcebe: Blood Centre, Hacettepe University, Ankara, Turkey

\section{Introduction}

Transfusions are life-saving in many conditions such as organ failure, injuries by physical trauma, bleeding for numerous reasons, with a certain risk for transmitting infectious agents or some diseases [1]. To decrease the risk of blood transmitted infections, blood donors generally go through a screening process including a miniphysical and/or serological examination and a questionnaire about general health status, medications used, and habitual behaviors for their own and the recipient's safety [2]. Although more improved detection, screening and inactivation techniques are used and current blood supply is safer than before, the safety of transfusion still depends on the selection of low risk donors [3]. Safety of blood and blood products is a highly concerned issue for all organizations. Except for institutions and university hospitals that have the capacity to handle possible transfusion problems, most hospitals still rely on blood suppliers for product safety and focus on patient safety [4]. These centers must be reliable for all aspects of collection, screening, testing and blood storage. Conventional safety measures are generally maintained by appropriate biomarkers for prevention of possible contamination via transfusion and for the health of not only receivers but also donors. If any problem is detected by these biomarkers, direct further clinical examination should be suggested. Some common tests for the detection of biological contaminants such as HIV and hepatitis viruses have been performed in blood centers so far [1]. As there are various different pathogens which cannot be screened by this strategy, anamnesis of donors should be detailed enough to fill this gap at least partly. It should be considered that these tests may be expensive and/or late markers, meaning these parameters can be detected after the symptoms of disorder occur.

In this regard, early and sensitive markers are strongly needed. Not only infections but also autoimmune processes and certain malignant diseases lead to an activation of the cellular immune system even if 
the diseases are still in an asymptomatic period. Neopterin is released from monocytes/macrophages with interferon $\gamma($ IFN- $\gamma)$ stimulation, and it has a potential function in the process of inflammation and infection as a mediator and/or regulator [1, 5-7]. Neopterin is also a nonspecific marker in many systemic problems, and the determination of neopterin levels in serum or urine would be useful to detect certain risk factors for blood transfusions [1].

Cellular immune activation also induces indoleamine-2,3-dioxygenase (IDO) in addition to neopterin release. Together with tryptophan-2,3-dioxygenase (TDO), IDO catalyzes the rate limiting step in the kynurenine pathway, in which tryptophan is degraded to kynurenine derivatives and nicotinamide adenine dinucleotides. Unlike TDO, IDO is preferentially induced by Th1-type cytokine IFN- $\gamma[8,9]$. The kynurenine-to-tryptophan (kyn/trp) ratio, that is, the ratio of the concentration of the first product of TDO and IDO versus the concentration of their substrate is an appropriate indicator of tryptophan degradation. The kyn/trp ratio provides a better and normalized measurement than absolute tryptophan or kynurenine concentrations. To substantiate that tryptophan degradation is due to the activation of IDO rather than TDO, it is necessary to demonstrate concomitant immune system activation. Thus, activated IDO is indicated when $\mathrm{kyn} / \operatorname{trp}$ correlates with an immune activation parameter and endogenous IFN- $\gamma$ formation [8-11]. The kyn/trp ratio generally correlates well with neopterin levels in accordance with progression and severity of diseases [11, 12]. Similar to disorders with increased neopterin production, increased degradation of tryptophan has also been described in some physiological conditions $[8,13,14]$.

The aim of this study was to evaluate neopterin levels and also tryptophan degradation in a group of Turkish blood donors. The effect of age, gender, blood type and $\mathrm{Rh}$ factor were also assessed.

\section{Materials and methods}

\section{Subjects and samples}

A total of 193 blood donors (149 males, 44 females; aged between 18 and 60 years) were included in the study. The study was approved by the local Ethics Committee of Hacettepe University in accordance with the Helsinki Declaration of 1981.

Samples of blood donors from the Blood Center of Hacettepe University were collected. Serum was obtained by centrifugation and all samples were stored at $-20^{\circ} \mathrm{C}$ until assayed.

\section{Measurements of neopterin}

Serum neopterin concentrations were measured by ELISA using a commercial kit (DRG, Marburg, Germany) according to the manufacturer's instructions. Results were expressed as nmol/L.

\section{Measurements of tryptophan/kynurenine concentrations and IDO activity}

Serum tryptophan and serum kynurenine concentrations were determined by high performance liquid chromatography (HPLC) [15] with minor modifications. The kyn/trp ratio was calculated to estimate IDO activity and expressed as $\mu \mathrm{mol} / \mathrm{mmol}$.

\section{Statistics}

Demographics are presented as mean \pm SD. All other results are presented as means \pm SEM. The correlations of the parameters were detected by non-parametric Spearman's correlation test. With Bonferroni correction, $\mathrm{p}<0.025$ and $\mathrm{p}<0.0125$ were considered statistically significant for two and four parameter correlations, respectively.

\section{Results}

Neopterin, tryptophan, kynurenine and kyn/trp levels of blood donors are presented in Table 1. Tryptophan, kynurenine and kyn/trp levels were found to be higher in males compared with females (all $\mathrm{p}<0.05$ ). Blood donors were divided into subgroups according to gender, age, smoking habit, Rh factor and blood types. The mean levels of measured parameters for each subgroup were established and evaluated (Table 1). In comparison with the 18-27 years age group, kynurenine levels were statistically higher in the 38-47 years and 48-60 years age groups; neopterin levels were lower in the $28-37$ years and 38-47 years age groups; and kyn/trp levels were higher in the 38-47 years and 48-60 years age groups (all $\mathrm{p}<0.05$ ). On the contrary; smoking, Rh (+/-) factor and blood types were all observed to have no effect on measured parameters (all $\mathrm{p}>0.05$ ).

The correlations between age and measured parameters were assessed. Age was found to be correlated with all measured parameters except for tryptophan (all $\mathrm{p}<0.05$ ). The relations of measured parameters were further investigated not only in donors as a whole but also in subgroups. These correlations are presented in Table 2.

The number and percentage of donors exceeding physiological levels of measured parameters were also analyzed. The levels were obtained from the manufacturer's 
Table 1 Characteristics of donors and measured parameters.

\begin{tabular}{|c|c|c|c|c|c|}
\hline \multirow[t]{2}{*}{ Groups } & \multirow[t]{2}{*}{$\mathbf{n}$} & \multicolumn{4}{|r|}{ Mean \pm SEM (min-max) } \\
\hline & & Trp & Kyn & Kyn/Trp & Neop \\
\hline \multicolumn{6}{|l|}{ Gender } \\
\hline Male & 149 & $66.0 \pm 0.9(40.4-100.6)$ & $2.5 \pm 0.05(1.2-4.2)$ & $38.0 \pm 0.8(21.2-76.4)$ & $7.9 \pm 0.3(3.0-22.6)$ \\
\hline Female & 44 & $60.5 \pm 2.0^{\mathrm{a}}(17.8-82.0)$ & $2.1 \pm 0.1^{\mathrm{a}}(0.4-3.5)$ & $34.5 \pm 1.5^{\mathrm{a}}(17.2-57.4)$ & $8.4 \pm 0.9(2.8-36.8)$ \\
\hline \multicolumn{6}{|c|}{ Age subgroup, years } \\
\hline $18-27$ & 64 & $66.7 \pm 1.4(42.9-94.7)$ & $2.2 \pm 0.06(1.4-3.7)$ & $34.2 \pm 1.1(22.0-61.3)$ & $7.1 \pm 0.5(2.8-20.3)$ \\
\hline $28-37$ & 60 & $63.8 \pm 1.8(17.8-100.6)$ & $2.4 \pm 0.1(0.4-3.9)$ & $37.2 \pm 1.4(17.2-68.0)$ & $8.1 \pm 0.5^{b}(3.1-18.3)$ \\
\hline $38-47$ & 52 & $65.2 \pm 1.4(47.4-100.6)$ & $2.5 \pm 0.1^{\mathrm{b}}(1.5-4.2)$ & $39.1 \pm 1.3^{b}(23.1-67.2)$ & $9.2 \pm 0.8^{\mathrm{b}}(3.5-36.8)$ \\
\hline $48-60$ & 17 & $63.2 \pm 3.1(45.3-87.3)$ & $2.6 \pm 0.2^{b}(1.3-4.1)$ & $41.6 \pm 2.8^{b}(26.2-76.4)$ & $7.5 \pm 0.7(3.8-15.4)$ \\
\hline \multicolumn{6}{|l|}{ Smoking } \\
\hline No & 93 & $65.1 \pm 1.3(17.8-100.6)$ & $2.4 \pm 0.07(0.4-4.2)$ & $37.0 \pm 1.0(17.2-76.4)$ & $7.9 \pm 0.4(2.8-20.3)$ \\
\hline Yes & 100 & $64.5 \pm 1.1(42.9-100.6)$ & $2.4 \pm 0.06(1.2-3.9)$ & $37.3 \pm 1.02(21.2-68.0)$ & $8.2 \pm 0.5(3.0-36.8)$ \\
\hline \multicolumn{6}{|l|}{ Rh factor } \\
\hline+ & 175 & $64.7 \pm 0.9(17.8-100.6)$ & $2.4 \pm 0.1(0.4-4.2)$ & $36.9 \pm 0.8(17.2-76.4)$ & $8.1 \pm 0.3(2.8-36.8)$ \\
\hline- & 18 & $65.6 \pm 2.1(52.3-87.1)$ & $2.6 \pm 0.1(1.6-3.6)$ & $40.0 \pm 2.5(26.6-67.2)$ & $7.3 \pm 0.7(3.8-16.1)$ \\
\hline \multicolumn{6}{|l|}{ Blood types } \\
\hline A Rh (+/-) & $81 / 6$ & $65.3 \pm 1.2(42.9-100.6)$ & $2.5 \pm 0.1(1.3-4.2)$ & $38.2 \pm 1.1(23.1-76.4)$ & $8.4 \pm 0.5(3.2-22.6)$ \\
\hline $\mathrm{B} \mathrm{Rh}(+/-)$ & $28 / 4$ & $63.3 \pm 2.6(17.8-100.6)$ & $2.2 \pm 0.1(0.4-3.5)$ & $34.9 \pm 1.6(21.2-54.1)$ & $6.6 \pm 0.5(2.8-14.5)$ \\
\hline $\mathrm{AB} \operatorname{Rh}(+/-)$ & $16 / 2$ & $67.8 \pm 1.9(57.1-80.2)$ & $2.2 \pm 0.1(1.5-3.2)$ & $33.4 \pm 1.9(20.2-47.5)$ & $8.1 \pm 0.7(3.5-15.0)$ \\
\hline $0 \mathrm{Rh}(+/-)$ & $50 / 6$ & $63.8 \pm 1.6(23.2-89.2)$ & $2.4 \pm 0.1(0.4-3.8)$ & $38.1 \pm 1.4(17.2-67.2)$ & $8.2 \pm 0.6(3.0-36.8)$ \\
\hline Total & 193 & $64.8 \pm 0.9$ & $4.7 \pm 0.1$ & $37.2 \pm 0.7$ & $8.0 \pm 0.3$ \\
\hline
\end{tabular}

Trp, tryptophan ( $\mu \mathrm{mol} / \mathrm{L}) ;$ Kyn, kynurenine ( $\mu \mathrm{mol} / \mathrm{L})$; Kyn/Trp ( $\mu \mathrm{mol} / \mathrm{mmol})$; Neop, neopterin $(\mathrm{nmol} / \mathrm{L}) ;{ }^{\mathrm{a}} \mathrm{p}<0.05$, compared with males; ${ }^{\mathrm{b}} \mathrm{p}<0.05$, compared with the $18-27$ years age group.

Table 2 Correlations between measured parameters in blood donors (total, $n=193$ ).

\begin{tabular}{|c|c|c|c|c|}
\hline \multirow[t]{2}{*}{ Groups } & \multirow[b]{2}{*}{ Neop-Trp } & \multicolumn{3}{|c|}{ Correlations (R-values and p-values) } \\
\hline & & Neop-Kyn & Neop-Kyn/Trp & Trp-Kyn \\
\hline All participants & $-0.076(0.293)$ & $0.361(<0.001)^{\mathrm{a}}$ & $0.399(<0.001)^{\mathrm{a}}$ & $0.266(<0.001)^{2}$ \\
\hline \multicolumn{5}{|l|}{ Gender } \\
\hline Male & $-0.041(0.621)$ & $0.380(<0.001)^{\mathrm{a}}$ & $0.370(<0.001)^{\mathrm{a}}$ & $0.227(0.005)^{a}$ \\
\hline Female & $-0.151(0.327)$ & $0.344(0.022)^{\mathrm{a}}$ & $0.480(0.001)^{\mathrm{a}}$ & $0.319(0.035)$ \\
\hline \multicolumn{5}{|l|}{ Age, years } \\
\hline $18-27$ & $0.098(0.442)$ & $0.254(0.043)$ & $0.181(0.153)$ & $0.256(0.042)$ \\
\hline $28-37$ & $0.101(0.445)$ & $0.579(<0.001)^{\mathrm{a}}$ & $0.541(<0.001)^{\mathrm{a}}$ & $0.397(0.002)^{a}$ \\
\hline $38-47$ & $-0.390(0.004)^{\mathrm{a}}$ & $0.093(0.513)$ & $0.346(0.012)^{\mathrm{a}}$ & $0.205(0.145)$ \\
\hline $48-60$ & $-0.181(0.486)$ & $0.132(0.613)$ & $0.331(0.195)$ & $0.520(0.033)$ \\
\hline \multicolumn{5}{|l|}{ Smoking } \\
\hline No & $0.049(0.638)$ & $0.341(0.001)^{\mathrm{a}}$ & $0.307(0.003)^{\mathrm{a}}$ & $0.271(0.009)^{\circ}$ \\
\hline Yes & $-0.189(0.060)$ & $0.360(<0.001)^{\mathrm{a}}$ & $0.456(<0.001)^{\mathrm{a}}$ & $0.228(0.022)^{a}$ \\
\hline \multicolumn{5}{|l|}{ Rh factor } \\
\hline+ & $-0.075(0.326)$ & $0.374(<0.001)^{\mathrm{a}}$ & $0.408(<0.001)^{\mathrm{a}}$ & $0.270(<0.001)^{2}$ \\
\hline- & $-0.147(0.561)$ & $0.113(0.656)$ & $0.190(<0.450)$ & $0.131(<0.604)$ \\
\hline \multicolumn{5}{|l|}{ Blood types } \\
\hline A Rh (+/-) & $-0.129(0.234)$ & $0.317(0.003)^{\mathrm{a}}$ & $0.354(0.001)^{\mathrm{a}}$ & $0.282(0.008)$ \\
\hline B Rh (+/-) & $-0.010(0.956)$ & $0.527(0.002)^{\mathrm{a}}$ & $0.517(0.002)^{\mathrm{a}}$ & $0.313(0.081)$ \\
\hline $\mathrm{AB} \operatorname{Rh}(+/-)$ & $-0.099(0.469)$ & $0.283(0.034)$ & $0.434(0.001)^{\mathrm{a}}$ & $0.247(0.066)$ \\
\hline $0 \mathrm{Rh}(+/-)$ & $0.207(0.411)$ & $0.228(0.362)$ & $0.221(0.378)$ & $0.018(0.945)$ \\
\hline
\end{tabular}

Neop, neopterin; Kyn/Trp, kynurenine-to-tryptophan ratio; ${ }^{\mathrm{a}} \mathrm{p}<0.05$. 
kit manual and previous publications [6, 8, 16, 17]. Out of 193 donors recruited in the study, 5 (2.6\%) had neopterin levels above $19 \mathrm{nmol} / \mathrm{L}, 22$ (11.4\%) had tryptophan levels over $78 \mu \mathrm{mol} / \mathrm{L}, 75$ (38.9\%) had kynurenine levels above $2.5 \mu \mathrm{mol} / \mathrm{L}$ and 99 (51.3\%) had kyn/trp levels exceeding $35 \mu \mathrm{mol} / \mathrm{mmol}$.

\section{Discussion}

Neopterin, playing a key role in immune activation, has become a valuable clinical parameter especially in infectious diseases, autoimmunity, malignant tumors, organ transplantations, and neurological and cardiovascular diseases as its measurement in body fluids is easy, fast and reproducible [16, 18-20]. Although donor questionnaires and routine screening tests are performed in blood transfusions, it has not yet been possible to clear transfusion-related infections. It has been shown that blood transfusion safety can be increased by screening neopterin, which is an early and sensitive marker of cellular immunity, in blood donors $[16,19]$. The release of neopterin begins 3 days before $\mathrm{T}$ cell proliferation reaches maximum, and an increase in neopterin production can be observed approximately 1 week before the appearance of specific antibodies [17, 21].

Neopterin has a distinct importance in blood banking as its levels increase in the incubation period of viral diseases before symptoms are observed [1, 6, 18, 19, 22]. The first study on this subject was performed in Austria, in the Innsbruck Blood Centre by Honlinger and colleagues [23]. Between 1986 and 1988, they determined neopterin levels of a huge number of blood donors in addition to routine tests. The 4-week follow-up of donors having yet high neopterin values resulted in the development of infectious and malignant diseases (57\%). On the basis of this data and further studies, neopterin is being screened in all Austrian blood banks since 1993 and bloods having high neopterin are not used for transfusion even if the other markers are negative [23]. In a retrospective study, Schennach and colleagues have reported that HCV-PCR positivity and high neopterin levels were correlated and screening neopterin levels in blood donations were helpful in decreasing infection risk [19].

IFN- $\gamma$ increases neopterin release in monocytes and macrophages and it also induces IDO, which plays an important role in tryptophan degradation via the kynurenine pathway. Therefore, in parallel with increased neopterin production, tryptophan breakdown increases in diseases related to cellular immune activation. Measurement of neopterin levels and kyn/trp enable the determination of the degree of immune activation and support each other [12, 22]. When two gender groups were compared, females were found to have statistically higher neopterin and lower kyn/trp levels. These changes were in accordance with previous studies and studies linking the difference in kyn/trp to hormonal status of gender [8, 20]. By contrast, there are studies indicating that neopterin does not change with gender [24].

The upper limit for serum tryptophan level in adults was considered to be $73 \pm 15 \mu \mathrm{mol} / \mathrm{L}$ [8]. In the study group, $11.4 \%(n=22)$ of tryptophan levels were high. The normal range for kynurenine levels was accepted to be $1.92 \pm 0.58$ $\mu \mathrm{mol} / \mathrm{L}[8]$. In this study, kynurenine levels were lower than the upper limit in 118 donors (61.1\%) and high in 75 donors (38.9\%). The mean of the high kynurenine group was detected as $>2.5 \mu \mathrm{mol} / \mathrm{L}$. When the upper limit of $\mathrm{kyn} / \mathrm{trp}$ was set as $26.9 \pm 8.1 \mu \mathrm{mol} / \mathrm{mmol}, 99(51.3 \%)$ donors had high kyn/trp levels [8, 25].

Serum neopterin levels may differ according to the manufacturer of ELISA kits. Normal neopterin concentration in healthy human serum differs according to the sources and analysts. For example, there are differences especially in estimating neopterin concentration at different ages and genders [1, 24]. Nevertheless, it is recommended to estimate its own neopterin referential values in each laboratory which conducts these assays. The average neopterin levels in this population show almost two-fold increases regarding various reports using different manufacturers of ELISAs. As our results are very different from other reports and need to be discussed with a different analysis, we had to define an individual cut-off point for this population with current results. This study provides a reference value for neopterin concentrations with $19.0 \mathrm{nmol} / \mathrm{L}$ as the upper limit. Regarding the manufacturer's kit manual, values obtained from the literature and our previous pteridine studies, normal levels of neopterin in adults are found to range between 3.7 and $19 \mathrm{nmol} / \mathrm{L}$ $[24,26,27]$. Concerning these lower and upper limits, in all participants $97.4 \%$ had neopterin levels in the normal range and $2.6 \%$ had high neopterin levels. In a study performed in the same center in 2005 by Fisenk et al., the prevalence of elevated neopterin levels was found to be $5.1 \%$ [6]. If these results are extrapolated to country, it can be considered that $2.5 \%$ of donations could not be used for blood transfusion. Regarding the fact that blood donation is not very common and the public are not aware of the importance of transfusion, this ratio is an important loss.

The effect of age on measured parameters was also investigated. Although there is no subject over 65 years of age in our study, in accordance with Frick et al. [25], it 
was observed that age and tryptophan levels were negatively correlated, whereas all other measured parameters were positively correlated. Neopterin levels were shown to change with age despite conflicting results $[17,25$, 28-30].

Pertovaara et al. have reported that IDO activity was lower in smokers compared with non-smokers [31]. Similarly, neopterin concentrations were also reported to be lower in smokers than non-smokers [16]. However, in the present study, smoking was found to have no effect on neopterin, tryptophan, kynurenine levels and IDO activity. This may result from the relatively small number of participants.

Murr et al. have investigated neopterin status in a broad donor group and found that neopterin levels were higher in O blood type compared with A, B and AB blood types [32]. In a study performed in Turkey, 17 donors with elevated neopterin levels were blood types $\mathrm{O}$ (7 donors), A (7 donors) and B (3 donors) (A. Yağmuroğlu, unpublished). In our study, the donors with the five highest neopterin concentrations were $\mathrm{O}$ (1 donor) and A (4 donors) blood type.

\section{References}

1. Schennach H, Murr C, Gachter E, Mayersbach P, Schonitzer D, Fuchs D. Factors influencing serum neopterin concentrations in a population of blood donors. Clin Chem 2002;48:643-5.

2. Wehrli G. Blood banking and transfusion medicine for the nephrologist. Semin Dial 2012;25:114-8.

3. Lindholm PF, Annen K, Ramsey G. Approaches to minimize infection risk in blood banking and transfusion practice. Infect Disord Drug Targets 2011;11:45-56.

4. Slapak C, Fredrich N, Wagner J. Transfusion safety: is this the business of blood centers? Transfusion 2011;51:2767-71.

5. Berdowska A, Zwirska-Korczala K. Neopterin measurement in clinical diagnosis. J Clin Pharm Ther 2001;26:319-29.

6. Fisenk BI, Us D, Ozcebe OI, Hascelik G. The value of increased neopterin levels in reducing transfusion-transmitted virus infections: detection of a donation from a HBSAg positive chronic carrier by screening of neopterin in Turkish blood donors. Scand J Infect Dis 2005;37:599-604.

7. Honlinger M, Fuchs D, Reibnegger G, Schonitzer D, Dierich MP, Wachter H. Neopterin screening and acute cytomegalovirus infections in blood-donors. Clin Invest 1992;70:63.

8. Schrocksnadel K, Wirleitner B, Winkler C, Fuchs D. Monitoring tryptophan metabolism in chronic immune activation. Clin Chim Acta 2006;364:82-90.

9. Munn DH, Shafizadeh E, Attwood JT, Bondarev I, Pashine A, Mellor AL. Inhibition of T cell proliferation by macrophage tryptophan catabolism. J Exp Med 1999;189:1363-72.

10. Widner B, Laich A, Sperner-Unterweger B, Ledochowski M, Fuchs D. Neopterin production, tryptophan degradation, and
Early and safe biomarkers are needed to determine possible changes in the organism, especially in routine analysis. Biomarkers are believed to decrease and even prevent primary and/or secondary health problems and economic loss. To increase the safety of blood transfusion, routine neopterin screening will be more economic and less time consuming. With neopterin screening, transfusions can be safer as it is possible to detect various infections that cannot be detected with serological tests such as mutant bacterial infections, pseudo-negative results, technical or staff errors.

In conclusion, as neopterin screening tests such as ELISA are cheaper and do not need an experienced technician, it is recommended to have routine neopterin screening in blood banks. In this regard, early diagnosis and medication will be possible and further health expenses will be prevented. Thus, applying neopterin analysis in blood banks in parallel with routine screening tests will help to reduce infection transmissions and increase safer transfusions.

Received March 25, 2013; accepted March 29, 2013; previously published online May 11, 2013

mental depression - what is the link? Brain Behav Immun 2002;16:590-5.

11. Taylor MW, Feng GS. Relationship between interferon-gamma, indoleamine 2,3-dioxygenase, and tryptophan catabolism. FASEB J 1991;5:2516-22.

12. Fuchs D, Moller AA, Reibnegger G, Werner ER, Werner-Felmayer $G$, Dierich MP, et al. Increased endogenous interferon-gamma and neopterin correlate with increased degradation of tryptophan in human immunodeficiency virus type 1 infection. Immunol Lett 1991;28:207-11.

13. Richard DM, Dawes MA, Mathias CW, Acheson A, Hill-Kapturczak N, Dougherty DM. L-Tryptophan: basic metabolic functions, behavioral research and therapeutic indications. Int J Tryptophan Res 2009;2:45-60.

14. Russo S, Kema IP, Fokkema MR, Boon JC, Willemse PH, de Vries EG, et al. Tryptophan as a link between psychopathology and somatic states. J Psychosom Med 2003;65: 665-71.

15. Widner B, Werner ER, Schennach H, Wachter H, Fuchs D. Simultaneous measurement of serum tryptophan and kynurenine by HPLC. Clin Chem 1997;43:2424-6.

16. Schennach H, Murr C, Gachter E, Mayersbach P, Schonitzer D, Fuchs D. Association between neopterin production and other parameters in a population of blood donor. Pteridines 2002;13:133-9.

17. Wachter H, Fuchs D, Hausen A, Reibnegger G, Weiss G, Werner ER, et al. Neopterin: biochemistry, methods, clinical application. Berlin/New York: Walter de Gruyter, 1992. 
18. Fuchs D, Wachter H, Schonitzer D. Neopterin and blood transfusion. Dtsch Med Wochenschr 1992;117:1578-9.

19. Schennach H, Hessenberger G, Mayersbach P, Schönitzer D, Fuchs D. Acute cytomegalovirus infections in blood donors are indicated by increased serum neopterin concentrations. Med Microbiol Immunol 2002;191:115-8.

20. Grebe SO, Mueller TF. Immune monitoring in organ transplantation using neopterin. Curr Drug Metab 2002;3:189-202.

21. Hoffmann G, Schobersberger W. Neopterin: a mediator of the cellular immune system. Pteridines 2004;15:107-12.

22. Hoffmann G, Wirleitner B, Fuchs D. Potential role of immune system activation-associated production of neopterin derivatives in humans. Inflamm Res 2003;52:313-21.

23. Honlinger M, Fuchs D, Hausen A, Reibnegger G, Schonitzer $D$, Werner ER, et al. Serum neopterin determination for the additional safeguarding of blood transfusions. Our experiences with 76,587 blood donors. Dtsch Med Wochenschr 1989;114:172-6.

24. Baydar T, Girgin G, Fuchs D, Inanici F, Sipahi H, Erol O, et al. Pteridine pathway in patients with degenerative diseases during short time treatment with low dose of meloxicam, as a non-steroidal anti-inflammatory drug. Pteridines 2008;19:107-13.

25. Frick B, Schroecksnadel K, Neurauter G, Leblhuber F, Fuchs $D$. Increasing production of homocysteine and neopterin and degradation of tryptophan with older age. Clin Biochem 2004;37:684-7.
26. Sarac ES, Girgin G, Palabiyik SS, Charehsaz M, Aydin A, Sahin $\mathrm{G}$, et al. A pilot study on neopterin levels and tryptophan degradation in zinc-exposed galvanization workers. Biol Trace Elem Res 2013;151:330-4.

27. Altindag ZZ, Baydar T, Isimer A, Sahin G. Neopterin as a new biomarker for the evaluation of occupational exposure to silica. Int Arch Occup Environ Health 2003;76:318-22.

28. Werner ER, Bichler A, Daxenbichler G, Fuchs D, Fuith LC, Hausen A. Determination of neopterin in serum and urine. Clin Chem 1987;33:62-6.

29. Frick B, Neurauter G, Diez-Ruiz A, Schroeksnadel K, Wirleitner $B$, Leblhuber F, et al. Neopterin and oxidation products in the blood of patients with various forms of dementia. Pteridines 2003;14:88-93.

30. Plata-Nazar K, Luczak G, Borkowska A, Delinska-Galinska A, Kozielska E, Marek K, et al. Reference standard of serum neopterin concentration in healthy children. Pteridines 2007;18:19-24.

31. Pertovaara M, Heliovaara M, Raitala A, Oja SS, Knekt P, Hurme $M$. The activity of the immunoregulatory enzyme indoleamine 2,3-dioxygenase is decreased in smokers. Clin Exp Immunol 2006;145:469-73.

32. Murr C, Schroecksnadel K, Schonitzer D, Fuchs D, Schennach H. Neopterin concentrations in blood donors differ between ABO blood group phenotypes. Clin Biochem 2005;38: 916-9. 